\title{
Antiviral activity of ISG15 against classical swine fever virus replication in porcine alveolar macrophages via inhibition of autophagy by ISGylating BECN1
}

Cheng Li ${ }^{1,2}$, Yifan Wang ${ }^{1}$, Hongqing Zheng ${ }^{1}$, Wang Dong ${ }^{3}$, Huifang LV ${ }^{3}$, Jihui Lin ${ }^{4}$, Kangkang Guo ${ }^{\text {* }}$ and Yanming Zhang ${ }^{1 *}$ (D)

\begin{abstract}
Interferons (IFNs) induce the expression of interferon-stimulated genes (ISGs) for defense against numerous viral infections, including classical swine fever virus (CSFV). However, the mechanisms underlying the effect of ISGs on CSFV infection are rarely reported. In this study, we demonstrate that IFN-a treatment induces upregulation of ISG15 and thus attenuates CSFV replication. To determine whether ISG15 is critical for controlling CSFV replication, we established porcine alveolar macrophages (PAMs) with stable overexpression or knockdown of ISG15. Overexpression of Flag-ISG15 significantly prevented CSFV replication, whereas loss of ISG15 led to abnormal proliferation of CSFV. Furthermore, upregulated ISG15 promoted beclin-1 (BECN1) ISGylation and dysfunction and subsequently inhibited autophagy, which is indispensable for CSFV replication. In addition, HECT and RLD domain containing E3 ubiquitin protein ligase 5 (HERC5), which functions to catalyze conjugation of ISG15 protein, was confirmed to interact with BECN1. Collectively, these results indicate that IFN-a restricts CSFV replication through ISG15-mediated BECN1 ISGylation and autophagy inhibition, providing insight into the mechanism of CSFV replication control by type I IFN. This mechanism may not be the only antiviral mechanism of ISG15; nonetheless, this study may contribute to the development of CSFV treatment and prevention strategies.
\end{abstract}

\section{Introduction}

Classical swine fever (CSF), which is caused by the CSF virus (CSFV) of family Flaviviridae, is a highly contagious viral disease of pig and has serious socioeconomic implications [1]. CSFV is an enveloped RNA virus with positive-sense genomic RNA and an open reading frame that encodes a precursor polyprotein of 3898 amino acids that are co- and post-translationally processed into four structural proteins $\left(C, E^{\text {rns }}, E 1, E 2\right)$ and eight

*Correspondence: Guokk2007@nwafu.edu.cn; zhangym@nwafu.edu.cn ${ }^{1}$ College of Veterinary Medicine, Northwest A\&F University, Yangling, Shaanxi, China

Full list of author information is available at the end of the article nonstructural proteins $\left(\mathrm{N}^{\mathrm{pro}}, \mathrm{p} 7, \mathrm{NS} 2, \mathrm{NS} 3, \mathrm{NS4A}, \mathrm{NS4B}\right.$, NS5A, NS5B) [2].

Type I interferon (IFN) has antiviral, antiproliferative, proapoptotic, and immunomodulatory activities. The production of type I IFN relies on the recognition of pathogen-associated molecular patterns by pattern recognition receptors, which induce an antiviral response by regulating synthesis of hundreds of proteins, inducing interferon-stimulated genes (ISGs) [3, 4]. RNA viruses of the family Flaviviridae are sensitive to type I IFN, and replication of CSFV is attenuated by treatment with IFN- $\alpha$ [5]. Although many studies have focused on screening and identifying antiviral ISGs and elucidating their antiviral mechanisms, only

c) The Author(s) 2020. This article is licensed under a Creative Commons Attribution 4.0 International License, which permits use, sharing, adaptation, distribution and reproduction in any medium or format, as long as you give appropriate credit to the original author(s) and the source, provide a link to the Creative Commons licence, and indicate if changes were made. The images or other third party material in this article are included in the article's Creative Commons licence, unless indicated otherwise in a credit line to the material. If material is not included in the article's Creative Commons licence and your intended use is not permitted by statutory regulation or exceeds the permitted use, you will need to obtain permission directly from the copyright holder. To view a copy of this licence, visit http://creativeco mmons.org/licenses/by/4.0/. The Creative Commons Public Domain Dedication waiver (http://creativecommons.org/publicdomain/ zero/1.0/) applies to the data made available in this article, unless otherwise stated in a credit line to the data. 
$2^{\prime}, 5^{\prime}$-oligoadenylate synthetase-like protein (OASL), myxovirus resistance protein 1 (Mx1), viperin, guanylate-binding protein 1 (GBP1), and IFN-inducible transmembrane proteins (IFITMs) have been identified to inhibit CSFV infection or replication [6-11].

Cells adapt to environmental changes through the initiation and execution of special processes, such as autophagy, which occurs in the event of poor survival conditions [12]. Autophagy processes include several stages, such as autophagy initiation signaling, autophagosome nucleation, autophagosome expansion, and autophagosome maturation [13]. It has been shown that CSFV infection triggers a complete autophagic response along with upregulation of autophagic markers, and induces formation of the membranes of autophagosome-like vesicles to promote viral replication in porcine kidney cells (PK-15) and porcine alveolar macrophages (PAMs) [14]. However, whether ISGs are involved in the regulation of autophagy during CSFV infection is largely unknown. Beclin-1 (BECN1), a highly conserved protein in mammals, is indispensable for canonical autophagy. BECN1 is a core component of the class III phosphatidylinositol 3-kinase (PI3KC3) complex, which is required for autophagosome nucleation and maturation. Furthermore, BECN1 regulates autophagic phosphatidylinositol 3-phosphate generation and recruits additional ATG proteins to orchestrate autophagosome formation [15].

ISG15 is one of the most abundantly induced ISGs and plays an important role in viral infection, but its role in regulating CSFV replication has not been extensively explored. ISG15 modifies its targets via a novel post-translational modification known as ISGylation, which spans a diverse array of biological processes and targets, including host and viral proteins [16]. Covalent conjugation of ISG15 to its target protein (ISGylation) is similar to ubiquitination and occurs through a sequential reaction catalyzed by UBE1L (E1)-activating, UbcH8 (E2)-conjugating, and HECT and RLD domain containing E3 ubiquitin protein ligase 5 (HERC5, E3) ligase enzymes [17]. ISGylation is a reversible process that requires ubiquitin-specific protease 18 (USP18) to remove ISG15 from the target protein [18]. Herein, we investigated the expression of ISG15 and ISGylation during CSFV infection. Experiments with shUSP18, ISG15 overexpression, and ISG15 mutant cells confirmed that the anti-CSFV action of ISG15 was ISGylation-dependent. BECN1 was ISGylated through ISG15 during CSFV infection, leading to autophagy-deficient CSFV reduction. Finally, BECN1 was demonstrated to interact with ISGylation E3 ligase.

\section{Materials and methods}

\section{Cells, medium, and virus}

The PAM cell line 3D4/2 [American Type Culture Collection (ATCC), Manassas, VA, USA; CRL-2845] was cultured in RPMI 1640 medium (Gibco, Grand Island, NY, USA) with $100 \mathrm{~mL} / \mathrm{L}$ (10\%) fetal bovine serum (Gibco). Human embryonic kidney (HEK-293T; ATCC) cells were cultured in Dulbecco's minimal essential medium (DMEM, Gibco) with 10\% fetal bovine serum (FBS, Gibco). IFN- $\alpha$ was purchased from Novoprotein (Shanghai, China), and 3-methyladenine (3MA) was purchased from Sigma-Aldrich (St. Louis, MO, USA). Rapamycin was obtained from Cell Signaling Technology (Boston, MA, USA). The Shimen strain of CSFV was purchased from the Control Institute of Veterinary Bio-products and Pharmaceuticals of China. All experiments related to CSFV were conducted in a P3 biosafety laboratory and strictly performed according to the Laboratory Biosafety Manual in the laboratory.

\section{Real-time quantitative polymerase chain reaction (RT-qPCR)}

The relative mRNA expression of ISGs and CSFV was detected by RT-qPCR. Primer pairs are listed in Table 1. Total RNA from PAMs was isolated using TRIzol (Thermo Fisher Scientific, Waltham, MA, USA) and reverse transcribed into cDNA using PrimeScript RT reagent kit (Vazyme, Nanjing, China). RT-qPCR was performed using Ultra SYBR Mixture (CWBIO, Beijing, China) according to the manufacturer's protocol. Relative fold changes in gene expression were normalized against $\beta$-actin expression using the $2^{-\Delta \Delta C t}$ threshold method.

\section{Western blot}

The protein samples of interest were separated by $12 \%$ sodium dodecyl sulfate-polyacrylamide gel

\section{Table 1 Primers used for RT-qPCR}

\begin{tabular}{lll}
\hline Primer & Sequence $\left(\mathbf{5}^{\prime} \mathbf{- 3} \mathbf{\prime}\right)$ & Usage \\
\hline ISG15-F & AGGGAACTGAAGGTGAAG & RT-qPCR for detection of ISG15 \\
& ATG & \\
ISG15-R & CAGACGCTGCTGGAAGG & \\
MX1-F & TCTGTAAGCAGGAGACCA & RT-qPCR for detection of MX1 \\
& TCAACT & \\
MX1-R & TTTCTCGCCACGTCCACTATC & \\
$\beta$-actin-F & CAAGGACCTCTACGCCAA & RT-qPCR for detection of \\
C-actin-R & TGGAGGCGCGATGATCTT & -actin \\
CSFV-F & GAGAAGGACAGCAGAACT & RT-qPCR for detection of CSFV \\
CSFV-R & ATAGC & \\
&
\end{tabular}


electrophoresis (SDS-PAGE) and transferred to polyvinylidene difluoride membranes (Millipore, Burlington, MA, USA). Following blocking in 5\% skim milk at room temperature for $2 \mathrm{~h}$, the membranes were immersed in the prepared primary antibody, which included mouse anti- $\beta$-actin monoclonal antibody (MAb) (Cell Signaling Technology), mouse anti-Myc MAb (Cell Signaling Technology), mouse anti-FLAG MAb (Sigma), mouse antiISG15 MAb (Cusabio, Wuhan, China), rabbit anti-LC3-II polyclonal antibody (PAb) (Sigma), and mouse antiBECN1 MAb (Sigma), and incubated at $4{ }^{\circ} \mathrm{C}$ overnight with shaking. After washing with Tris-buffered saline containing $0.5 \%$ Tween 20 (TBST), the membranes were incubated with horseradish peroxidase (HRP)-conjugated secondary antibodies at room temperature for $2 \mathrm{~h}$, followed by three washes in TBST. The protein bands were analyzed using an image analysis system (Bio-Rad) after incubation with enhanced chemiluminescence (ECL) reagent. $\beta$-actin served as an internal control.

\section{Construction and transfection of plasmid}

Based on the genetic sequences of porcine ISG15, USP18, HERC5, and BECN1 (GenBank: EU647216.1, NM_213826.1, XM_021100766.1, and NM_001044530.1), PCR primers for amplification were generated (Table 2). ISG15 was inserted into the lentivirus plasmid pCDH-CMV-MCS-EF1 (CMV) with a Flag-tag to generate CMV-ISG15 and was cloned into pcDNA3.1 with a Flag-tag to generate Flag-ISG15
(3.1-ISG15GG). Synthesis of 3.1-ISG15AA from 3.1-ISG15GG was performed by directed mutagenesis with a Phusion site-directed mutagenesis kit (Thermo Fisher Scientific) following the manufacturer's instructions. BECN1 was cloned into pcDNA3.1 or pGEX6p-1 to create Flag- or glutathione S-transferase (GST)-tagged BECN1, respectively. The N-terminus of $B E C N 1$ fused with the C-terminal sequence LRLRGG of ISG15 was inserted into pcDNA3.1 to generate Flagtagged ISG15-BECN1. HERC5 was amplified by PCR and inserted into pcDNA3.1 or pGEX-6p-1 to generate Myc- or GST-tagged HERC5. As for the short hairpin RNA (shRNA) plasmid, three pairs of shRNA for ISG15 or USP18, as well as scrambled control shRNA $(\mathrm{shN})$, were designed using the RNAi Designer website. All shRNAs were inserted into pCDH-U6-GreenPuro. The shRNA sequences are listed in Table 3. CMV, pcDNA3.1, and pCDH-U6-GreenPuro plasmids and pGAG, pREV, and pVSV-G helper plasmids were conserved in our laboratory. 3.1-Flag-RFP (RFP cloned into pcDNA3.1 with a Flag-tag) was constructed and conserved in our laboratory. To transiently transfect the plasmids into PAMs, a mixture containing $4 \mu \mathrm{g}$ plasmids, $6 \mu \mathrm{L}$ Turbofect (Thermo Fisher Scientific), and $400 \mu \mathrm{L}$ Opti-MEM was added to the PAMs and incubated for $8 \mathrm{~h}$. Then, the medium was refreshed with $10 \%$ FBS 1640 and incubated for another $24 \mathrm{~h}$ for subsequent experiments.

\section{Table 2 Primers used for plasmid construction}

\begin{tabular}{|c|c|c|}
\hline Primer & Sequence $\left(5^{\prime}-3^{\prime}\right)$ & Usage \\
\hline CMV-ISG15-F & CGGAATTCATGGGTAGGGAACTGAAGGTGAAGA & Amplification of ISG15 \\
\hline CMV-ISG15-R & CGGGATCCGCACTCGGTGGGGTGCTCCC & \\
\hline 3.1-ISG15AA-A2 & TGGCCCTGTCCCCGCCGCCCGCAGGCGCAG & Amplification of ISG15 mutant \\
\hline 3.1-ISG15AA-B1 & CTGCGCCTGCGGGCGGCGGGGACAGGGCCA & \\
\hline Flag-ISG15-F & CGGAATTCTATGGGTAGGGAACTGAAGGTGAAGA & Amplification of ISG15 \\
\hline Flag-ISG15-R & CGGGATCCGCACTCGGTGGGGTGCTCC & \\
\hline Myc-BECN1-F & CGGAATTCTATGGAGGGGTCTAAGACATCCA & Amplification of BECN1 \\
\hline Myc-BECN1-R & CGGGATCCTTTGTTATAAAACTGTGAGGATACCC & \\
\hline Flag-BECN1-F & CGGAATTCCATGGAGGGGTCTAAGACATCCA & Amplification of BECN1 \\
\hline Flag-BECN1-R & CGGGATCCTTTGTTATAAAACTGTGAGGATACCC & \\
\hline Flag-ISG15-BECN1-A2 & CTACAGAATCTGGGGAGGTAGCCCCCCCGCAGGCGCAGATT & Amplification of ISG15-BECN1 \\
\hline Flag-ISG15-BECN1-B1 & ATCTGCGCCTGCGGGGGGGCATGGAGGGGTCTAAGACATCCA & \\
\hline Myc-HERC5-F & TGC TCTAGA ATGGAGCGGAGACCACGGAG & Amplification of HERC5 \\
\hline Myc-HERC5-R & CG GGATCC GCCAAATCCCCTGTGGTTGC & \\
\hline GST-BECN1-F & CGGAATTCATGGAGGGGTCTAAGACATCCA & Amplification of BECN1 \\
\hline GST-BECN1-R & CGGGATCCTTTGTTATAAAACTGTGAGGATACCC & \\
\hline GST-HERC5-F & CG GGATCC ATGGAGCGGAGACCACGGAG & Amplification of HERC5 \\
\hline GST-HERC5-R & CCG CTCGAG TCAGCCAAATCCCCTGTGGTT & \\
\hline
\end{tabular}


Table 3 Short hairpin RNA (shRNA) sequences

\begin{tabular}{|c|c|}
\hline shRNA & Sequence $\left(5^{\prime}-3^{\prime}\right)$ \\
\hline shISG15-1-F & GATCCGACCAGTTCTGGCTGACTTTCCAAGAGGAAAGTCAGCCAGAACTGGTCTTTTTTG \\
\hline shISG15-1-R & AATTCAAAAAGACCAGTTCTGGCTGACTTTCCTCTTGGAAAGTCAGCCAGAACTGGTCG \\
\hline shISG15-2-F & GATCCGCCTATGTGCACCGTGTATATCAAGAGATATACACGGTGCACATAGGCTTTTTG \\
\hline shISG15-2-R & AATTCAAAAAGCCTATGTGCACCGTGTATATCTCTTGATATACACGGTGCACATAGGCG \\
\hline shISG15-3-F & GATCCGTGCACCGTGTATATGAATCTCAAGAGAGATTCATATACACGGTGCACTTTTTG \\
\hline shISG15-3-R & AATTCAAAAAGTGCACCGTGTATATGAATCTCTCTTGAGATTCATATACACGGTGCACG \\
\hline shUSP18-1-F & GATCCGGTCGGTTTGCACAACATTGGCAAGAGCCAATGTTGTGCAAACCGACCTTTTTG \\
\hline shUSP18-1-R & AATTCAAAAAGGTCGGTTTGCACAACATTGGCTCTTGCCAATGTTGTGCAAACCGACCG \\
\hline shUSP18-2-F & GATCCGCCTTAACTCCCTGATTCAGGCAAGAGCCTGAATCAGGGAGTTAAGGCTTTTTTG \\
\hline shUSP18-2-R & AATTCAAAAAGCCTTAACTCCCTGATTCAGGCTCTTGCCTGAATCAGGGAGTTAAGGCG \\
\hline shUSP18-3-F & GATCCGCCTACTGTCTCCAGAAGTACCAAGAGGTACTTCTGGAGACAGTAGGCTTTTTG \\
\hline shUSP18-3-R & AATTCAAAAAGCCTACTGTCTCCAGAAGTACCTCTTGGTACTTCTGGAGACAGTAGGCG \\
\hline
\end{tabular}

Underlines show loop ring.

\section{Cell viability assay}

The cell viability assay was performed with the Cell Counting Kit-8 (CCK-8) (Beyotime) according to the manufacturer's instructions.

\section{Establishment and detection of ISG-overexpression and -knockdown cell lines}

CMV plasmid containing ISG15 or pCDH-U6 plasmids carrying shISG15 or shUSP18 were co-transfected into HEK-293T cells with three ancillary plasmids (pGAG, pREV, and pVSV-G) by Turbofect. These cells were cultured in DMEM with 2\% FBS for $16 \mathrm{~h}$, and the medium was replaced with DMEM containing 10\% FBS, $0.01 \mathrm{mM}$ cholesterol (Sigma), $0.01 \mathrm{mM} \mathrm{L-} \alpha$-phosphatidylcholine (Sigma), 1:1000 diluted Chemically Defined Lipid (Invitrogen, Carlsbad, CA, USA), and $4.0 \mathrm{mM} \mathrm{L}$-glutamine (Invitrogen) followed by another $48 \mathrm{~h}$ of incubation. The supernatants were collected and centrifuged at $1500 \mathrm{~g}$ to obtain the 5 types of lentiviruses (CMV, CMV-ISG15, shN, shISG15, and shUSP18). Five lentiviruses were added to PAMs in a 6-well culture plate, respectively. The medium was exchanged with fresh medium at $8 \mathrm{~h}$ after infection of lentivirus, followed by incubation for another 48 h. CMV and shN served as negative controls. Puromycin (Thermo Fisher Scientific) was used to screen positive cells in complete growth medium at a final concentration of $2 \mu \mathrm{g} / \mathrm{mL}$. To observe the screened positive cells that exhibited green fluorescence, a fluorescence inversion microscope (Nikon, Tokyo, Japan) was used. Western blot was performed to confirm the overexpression or knockdown of ISGs.

\section{Virus titration by immunofluorescence assay (IFA)}

IFAs were performed to estimate the virus titers of CSFV in the cellular supernatant. PAMs were inoculated with cellular supernatant in a 96-well culture plate with 10 groups generated by a tenfold dilution series $\left(10^{-1}-10^{-10}\right)$ for $48 \mathrm{~h}$, and eight repetitions for each dilution were performed. A negative control was generated by culture with medium without CSFV. These cell samples were fixed by 1:1 fixing liquid (acetone:methanol) at $-20{ }^{\circ} \mathrm{C}$ for $20 \mathrm{~min}$ and permeabilized by $0.1 \%$ Triton $\mathrm{X}-100$ in a $4{ }^{\circ} \mathrm{C}$ refrigerator for $20 \mathrm{~min}$. Permeabilized cells were incubated with $5 \%$ skim milk for $2 \mathrm{~h}$ at room temperature, followed by incubation with positive CSFV serum conserved in our laboratory at $4{ }^{\circ} \mathrm{C}$ for about $16 \mathrm{~h}$. After washing, cells were incubated with fluorescein isothiocyanate (FITC)conjugated rabbit anti-pig IgG antibody (Sigma) for $2 \mathrm{~h}$. Between each step of the procedure, cells were washed three times using phosphate-buffered saline (PBS) or PBS with Tween 20 in the case of antibody washing. Negative controls were used for background subtraction. FITCpositive cells were observed and counted under a fluorescence inversion microscope (Nikon). The number of positive and negative wells of each dilution was recorded. The viral titers were expressed as $50 \%$ tissue culture infectious dose $\left(\mathrm{TCID}_{50}\right) / \mathrm{mL}$.

\section{Confocal immunofluorescence microscopy}

To analyze the effect of different treatment or transfection on autophagosomes, accumulation of LC3 puncta was quantified. Cells in 35-mm culture dishes were fixed by $1: 1$ fixing liquid (acetone:methanol) at $4{ }^{\circ} \mathrm{C}$ for $30 \mathrm{~min}$ following three washes with PBS for $10 \mathrm{~min}$ each. Then, the cell samples were permeabilized by $0.1 \%$ Triton X-100 at $4{ }^{\circ} \mathrm{C}$ for $20 \mathrm{~min}$ and blocked with $5 \%$ skim milk at room temperature for $2 \mathrm{~h}$. Cells were incubated with a rabbit anti-LC3 PAb (Sigma) as a primary antibody and Alexa Fluor 488 AffiniPure goat anti-rabbit IgG $(\mathrm{H}+\mathrm{L})$ antibody (Yeasen, Shanghai, China) as a secondary antibody. 
Nuclei were counterstained with 4',6-diamidino-2-phenylindole at room temperature for $10 \mathrm{~min}$ and imaged by laser scanning confocal microscopy (LSM510 META, Zeiss, Oberkochen, Germany). In most cases, $>12$ cells were analyzed for each set, and each experiment was repeated three times. The average amount of LC3 puncta per cell from each group was analyzed with Image J and is presented graphically.

\section{Transmission electron microscopy (TEM)}

PAMs were seeded in a 100-mm culture dish and treated with IFN- $\alpha$, rapamycin, or CSFV, while the mock group was incubated with an equivalent amount of 1640 medium. After $48 \mathrm{~h}$, the cells were collected in a $1.5-\mathrm{mL}$ Eppendorf tube, centrifuged at $1000 \mathrm{~g}$ at $4{ }^{\circ} \mathrm{C}$ for $10 \mathrm{~min}$, and then immobilized by $2.5 \%$ glutaraldehyde (Solarbio, Bejing, China). Fixed cells were sliced and observed with TEM.

\section{Co-immunoprecipitation (Co-IP) and GST pull-down assays} Flag-BECN1 and Myc-HERC5 plasmids were constructed for Co-IP assay, and irrelevant Flag-RFP served as a negative control. PAMs seeded in a 6-well culture plate were co-transfected with $2 \mu \mathrm{g}$ of Flag-BECN1 and Myc-HERC5 using Turbofect and cultured for $48 \mathrm{~h}$. After incubation, PAMs were collected and lysed by immunoprecipitation (IP) lysis buffer containing phenylmethanesulfonyl fluoride (Beyotime). The negative control was subjected to the same procedure. The protein supernatant was collected through centrifugation at $12000 \mathrm{~g}$ at $4{ }^{\circ} \mathrm{C}$ and used for Co-IP assay with Anti-Flag M2 Affinity Gel (Sigma) or Anti-c-Myc Agarose Affinity Gel (Sigma) in accordance with the manufacturer's instructions. After three washes with TBS, the immunoprecipitate was added to SDS-PAGE loading buffer and denatured for Western blot utilizing anti-Flag or anti-Myc antibodies.

To explore the direct interaction between BECN1 and HERC5, GST-BECN1 and GST were expressed in Escherichia coli BL21 cells, and Myc-HERC5 was expressed in HEK-293T cells. The GST pull-down tests were performed using the Pierce GST Protein Interaction Pull-Down Kit (Thermo Fisher Scientific) based on the manufacturer's instructions. Glutathione agarose resin slurry was added to each of the two Pierce spin columns, after which a mixture of $200 \mu \mathrm{L}$ TBS and $200 \mu \mathrm{L}$ pull-down lysate blend was added. The columns were centrifuged for $45 \mathrm{~s}$ at $1500 \mathrm{~g}$ four times to balance the glutathione agarose resin. GST and GST-BECN1 were incubated with two of the balanced glutathione agarose resin samples at $4{ }^{\circ} \mathrm{C}$ for $60 \mathrm{~min}$, followed by centrifugation and washing. Then, Myc-HERC5 was injected into the mixture and incubated at $4{ }^{\circ} \mathrm{C}$ for $2 \mathrm{~h}$. Finally, elution buffer containing $3.1 \mathrm{mg}$ GST per milliliter of TBS was used to separate the precipitate, which was centrifuged and boiled with SDS-PAGE loading buffer for $5 \mathrm{~min}$. The interaction between BECN1 and HERC5 in the samples was detected by Western blot assay with anti-Myc antibody. To further confirm the interaction, GST-HERC5 and Flag-BECN1 were evaluated with the same method, and the eluted proteins were detected by Western blot assay with anti-Flag antibody.

\section{Statistical analysis}

Data are presented as the mean \pm standard deviation (SD) of three independent experiments. Student's $t$-test was used for all statistical analyses. Differences in each group were considered to be significant with $P$ values less than 0.05 .

\section{Results \\ CSFV replication is restricted by ISG15 in PAMs}

To determine the role of ISG15 in CSFV infection, we constructed a cell line stably overexpressing Flag-ISG15 protein (CMV-ISG15 cells) by lentivirus. PAMs stably transfected with an empty vector CMV by lentivirus was regarded as a negative control (CMV cells). Overexpression of Flag-ISG15 in PAMs was easily detected by anti-Flag or anti-ISG15 antibody (Figure 1A) and did not affect cell proliferation and viability (Figure 1B). Western blot results showed that the efficiency of transfection was high (with anti-Flag antibody) and the exogenous expression of ISG15 was successful (with anti-ISG15 antibody). The expression of GFP indicated the efficiency of transfection. Green fluorescence was visualized in CMV and CMV-ISG15 cells under an inverted fluorescence microscope, but no green fluorescence was detected in the mock-transfected cells (Figure 1C). The positively transfected cell rate was approximately $100 \%$. Compared with the transcriptional levels of CSFV in PAMs or CMV cells, those in CMV-ISG15 cells were significantly inhibited at 12 hours post-infection (hpi) and $24 \mathrm{hpi}$, respectively. CSFV titers in culture supernatants of CMV-ISG15 cells significantly decreased compared with those in CMV cells at $12 \mathrm{hpi}$ and $24 \mathrm{hpi}$ (Figure 1D). These data suggested that overexpression of Flag-ISG15 inhibits CSFV replication in PAMs.

To explore the effect of endogenous ISG15 on CSFV propagation, three PAM cell lines stably tranfected with shISG15-1, shISG15-2 and shISG15-3 and a negative control (shN cells) mediated by lentivirus infection were constructed. ISG15 expression in shISG15-1 cells was decreased compared with that in shN cells, exhibiting the highest knockdown efficiency (Figure 1E), and did not affect cell proliferation and viability (Figure 1F). The expression of GFP was used to indicate the transfection efficiency. Green fluorescence was visualized in shN and 


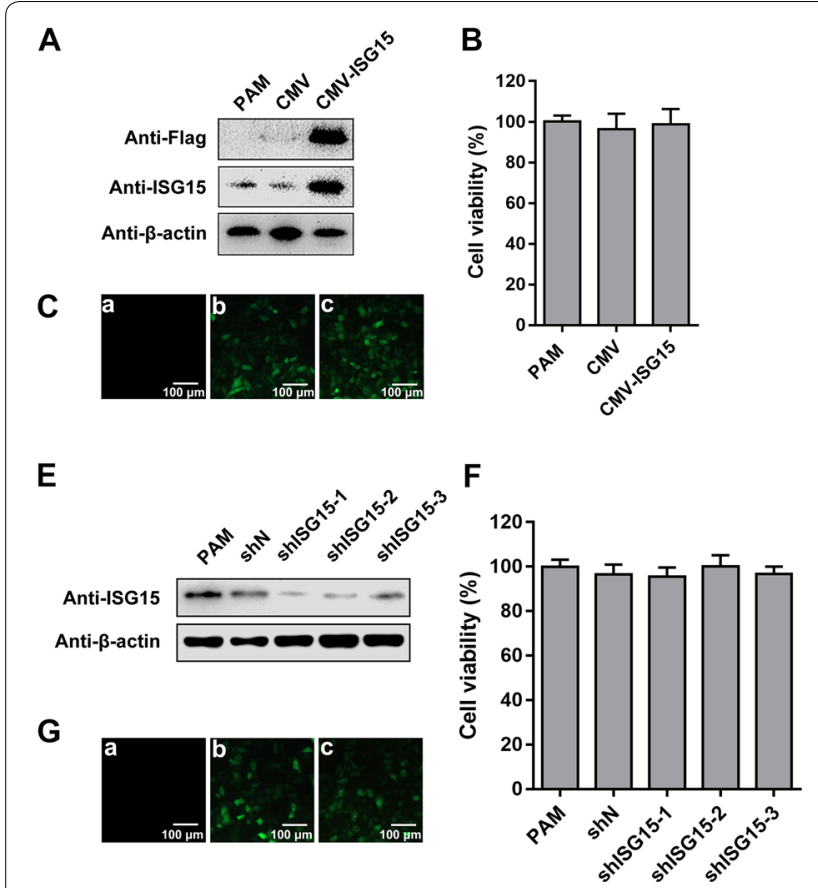

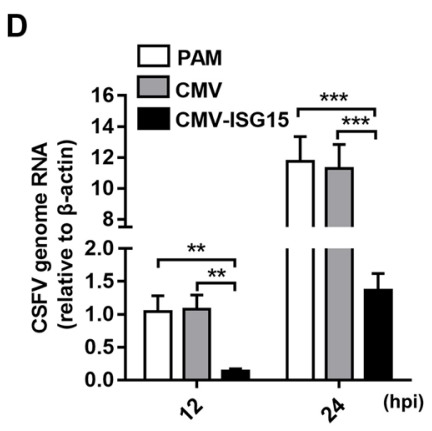

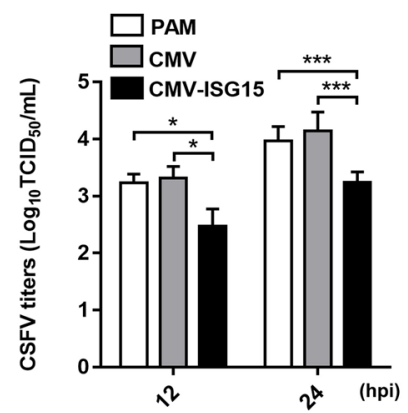

H

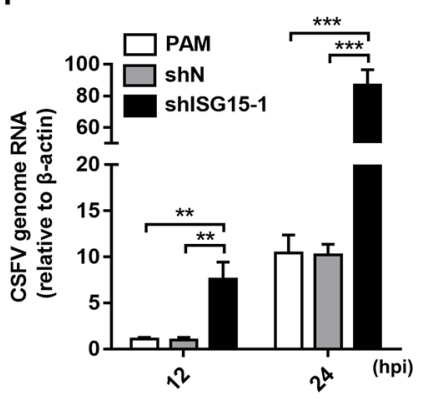

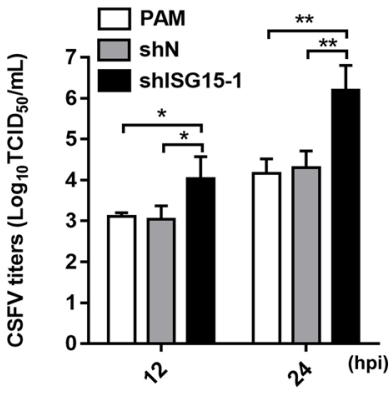

Figure 1 CSFV replication is restricted by ISG15 in PAMs. PAMs stably overexpressing ISG15 were constructed by lentiviral transduction. A Expression of ISG15 protein in PAMs and, CMV and CMV-ISG15 cells was determined by Western blot using an anti-Flag and an anti-ISG15 antibody, respectively. $\beta$-actin served as an internal control. B Cell viability of cell lines stably overexpressing ISG15. C GFP reporter expression was detected on mock-infected cells (a), CMV-infected cells (b) and I CMV-ISG15-infected cells (c). Scale bars, $100 \mu \mathrm{m}$. CSFV genomic RNA and viral titers D were analyzed in CMV and CMV-ISG15 cells at 12 and $24 \mathrm{hpi}(\mathrm{MOI}=1)$ by RT-qPCR and IFA, respectively. E Expression of ISG15 protein was probed by Western blot. F Cell viability of cell lines with stable knockdown of ISG15. G GFP reporter expression was detected in mock-infected PAMs (a), shN-infected cells (b) and shISG15-1-infected cells (c). Scale bars, $100 \mu \mathrm{m}$. ShN and shISG15 cells were incubated with CSFV (MOI=1). G CSFV genomic RNA was quantified in shN and shISG15 cells at 12 and 24 hpi. $\mathbf{H}$ The extracellular viral titers were quantified and expressed as TCID ${ }_{50} / \mathrm{mL}$. Data $(\mathbf{B}, \mathbf{D}, \mathbf{F}$, and $\mathbf{H})$ represent the mean $\pm S D$ of three independent experiments and were measured in technical duplicate. Comparisons between groups were calculated using Student's $t$-test. ${ }^{*} P<0.05$; ${ }^{* *} P<0.01$; ${ }^{* * *} P<0.001$

shISG15-1 cells under an inverted fluorescence microscope (Figure 1G). The rate of positively transfected cells was approximately $100 \%$. Thus, shISG15-1 cells, referred to as shISG15 cells hereafter, were used for subsequent experiments. PAMs and, shISG15 and shN cells were infected with CSFV (multiplicity of infection $[\mathrm{MOI}]=1$ ), and CSFV genomic RNA levels and the progeny virus titers were dramatically increased in shISG15 cells compared with those in PAMs or shN cells at both $12 \mathrm{hpi}$ and 24 hpi (Figure $1 \mathrm{H}$ ). These results demonstrated that ISG15 is essential for controlling CSFV propagation in PAMs.

\section{ISG15 is induced by IFN- $a$ and contributes to IFN antiviral activity against CSFV}

To confirm that ISG15 can be induced by type I IFN in PAMs, the cells were treated with different amounts of IFN- $\alpha$. As expected, the expression of ISG15 was rapidly and robustly induced by IFN- $\alpha$ in a dose-dependent manner, whereas the cell viability displayed no considerable changes (Figures 2A and B). Furthermore, ISG15 expression was highly induced at $12 \mathrm{~h}$ (43.24-fold), $36 \mathrm{~h}$ (56.14-fold), and $48 \mathrm{~h}$ (52.38-fold) and peaked at $24 \mathrm{~h}$ (69.99-fold) (Figure 2C). ShISG15 and shN cells were pretreated with IFN- $\alpha$ for $24 \mathrm{~h}$ as a positive control, followed by $\operatorname{CSFV}(\mathrm{MOI}=1)$ infection. As shown in Figures $2 \mathrm{D}$ and $E$, IFN- $\alpha$ pretreatment significantly increased ISG15 expression and inhibited CSFV replication in shN cells, whereas knockdown of ISG15 resulted in a lower response to IFN- $\alpha$ treatment and aberrant CSFV replication, indicating that ISG15 is indispensable for the IFN$\alpha$-mediated antiviral effect.

\section{ISG15 expression is upregulated in CSFV-infected PAMs} ISG15 expression of PAMs infected with CSFV $(\mathrm{MOI}=1)$ was analyzed at the indicated times postinfection. The results showed that ISG15 expression was significantly upregulated at $12,24,36$, and $48 \mathrm{hpi}$ compared with that in the mock PAMs at both the mRNA and protein levels (Figures 3A, B). Intriguingly, following infection with different doses of CSFV 

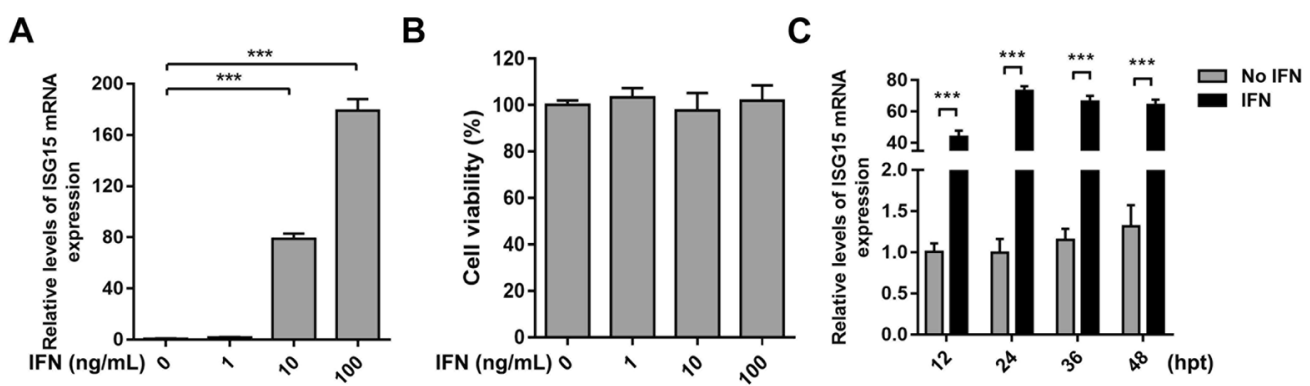

D

\section{E}
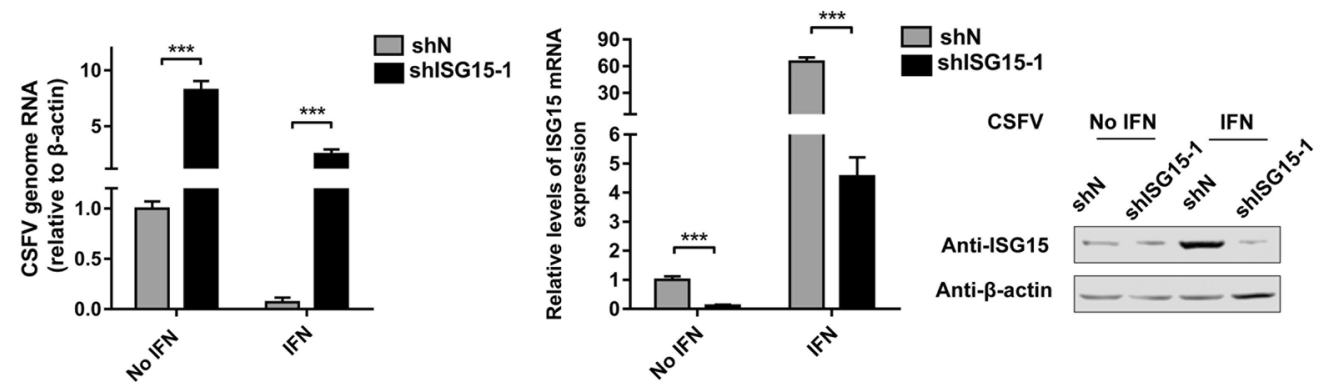

Figure 2 ISG15 is induced by IFN- $\boldsymbol{a}$ and contributes to IFN antiviral activity against CSFV. PAMs were treated with different amounts of IFN-a $(0,1,10$, and $100 \mathrm{ng} / \mathrm{mL})$ for $24 \mathrm{~h}$. A $/ S G 15$ expression was determined by RT-qPCR. B Cell viability was examined by MTT assay. C Mock- and IFN-a-treated (10 ng/mL) PAMs were harvested at the indicated times post-treatment. ISG15 expression was quantified by RT-qPCR. IFN-a (10 ng/ $\mathrm{mL})$ was added as a positive control. ShISG15 and shN cells were mock-treated or treated with IFN-a (10 ng/mL) for $24 \mathrm{~h}$ prior to CSFV infection $(\mathrm{MOI}=1)$. D CSFV genomic RNA was quantified by RT-qPCR. E ISG15 mRNA and protein levels were analyzed by RT-qPCR and Western blot. Data $(\mathbf{A}, \mathbf{B}, \mathbf{C}, \mathbf{D}$, and $\mathbf{E})$ represent the mean $\pm \mathrm{SD}$ of three independent experiments and were measured in technical duplicate. Comparisons between groups were calculated using Student's t-test. ${ }^{* * *} P<0.001$

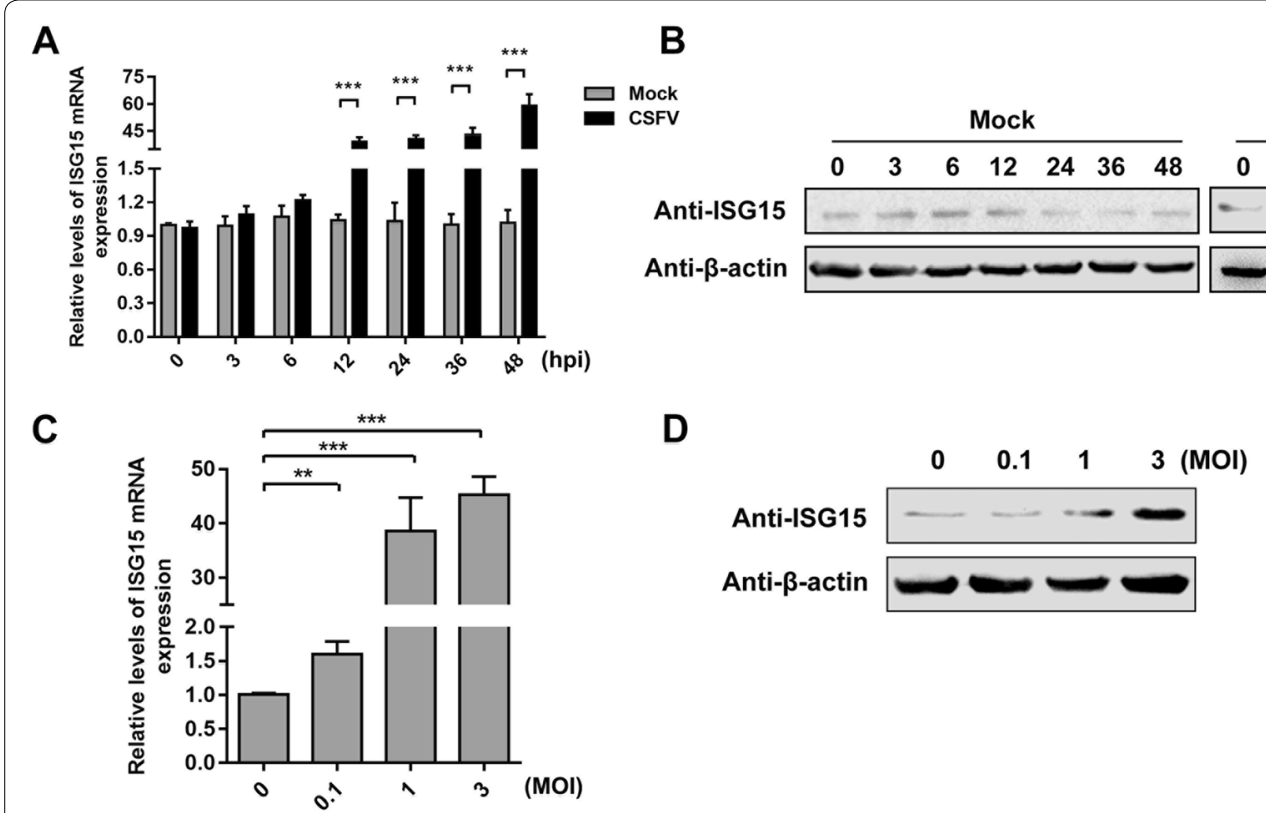

Figure 3 ISG15 expression is upregulated in CSFV-infected PAMs. A, B Expression of ISG15 in PAMs incubated with CSFV $(\mathrm{MOI}=1)$ at 0, 3, 6, 12, 24, 36, and 48 hpi. A ISG 15 expression was quantified by RT-qPCR. and Western blot. C, D Expression of ISG15 in PAMs incubated with CSFV $(\mathrm{MOI}=0.1,1$, and 3). The expression of ISG15 was quantified by RT-qPCR (C) and Western blot (D) at 24 hpi. $\beta$-actin served as an internal control. Data $(\mathbf{A}, \mathbf{C})$ represent the mean \pm SD of three independent experiments and were measured in technical duplicate. Comparisons between groups were calculated using Student's $t$-test. ${ }^{* *} P<0.01 ;{ }^{* * *} P<0.001$ 
$(\mathrm{MOI}=0.1,1$, or 3$)$ for $24 \mathrm{~h}$, both mRNA and protein levels of ISG15 were upregulated (Figures 3C, D).

\section{ISGylation is involved in the antiviral activity of ISG15 against CSFV}

To determine whether ISG15 exerts its anti-CSFV activity in a conjugated manner, a mutated form of ISG15 (3.1-ISG15AA) was constructed via substituting GG with AA by site-directed mutagenesis. In 3.1-ISG15AA-transfected cells, only the free ISG15 level increased compared with that in the control, whereas the ISGylation level did not increase as much as that in 3.1-ISG15GG-transfected cells, suggesting that 3.1-ISG15AA-transfected cells lost the ability to conjugate with the target protein (Figure 4A). PAMs transfected with 3.1 were used as a control. CSFV genomic RNA levels and viral titers in 3.1-ISG15GGtransfected PAMs were significantly decreased compared with those in 3.1-transfected PAMs (Figure 4B). To exclude the influence of basal levels of ISG15, similar experiments were performed in shISG15 cells, which yielded similar results (Figure $4 \mathrm{C}$ ). These results revealed that CSFV can be inhibited by conjugative ISG15. Furthermore, USP18, an ISG15-specific deconjugating protease that cleaves conjugation of ISG15, was knocked down in PAMs. We constructed cell lines stably transfected with USP18 shRNAs (shUSP18-1, shUSP18-2 and shUSP18-3) and a negative control (shN cells). The knockdown efficiency of shUSP18-2 was the highest at the protein level, and the viability of these cells was similar to that of mock-transduced PAMs which served as a control (Figures 4D, E). As shown in Figures 4F and G, compared with levels in the shN cells, knockdown of USP18 led to an upregulation of ISG15 conjugates and decrease in CSFV replication in IFNpretreated cells, indicating that USP18 deficiency promoted ISGylation to inhibit CSFV replication.

Because both ISG15 and USP18 regulate the type I IFN signaling pathway, we evaluated $M x 1$ (another ISG) expression in ISG15-knockdown cells and USP18knockdown cells following IFN- $\alpha$ pretreatment and subsequent infection with CSFV. As shown in Figure $4 \mathrm{H}$, knockdown of ISG15 expression upregulated $M x 1$ expression, and a similar result was obtained in USP18-knockdown cells. However, knockdown of ISG15 and knockdown of USP18 showed opposite effects on CSFV replication (Figures $2 \mathrm{H}$ and $3 \mathrm{H}$ ), suggesting that the effect of ISGylation was more pronounced than the effect of the IFN pathway in terms of inhibition of CSFV. These results suggested that ISG15 can impair CSFV replication in an ISGylation-dependent manner.

\section{ISG15 inhibits autophagy, which is required for CSFV replication}

To test whether ISG15 interferes with CSFV-induced autophagy, protein expression of LC3 and the number of fluorescent puncta of LC3 were assessed in ISG15-overexpressing and ISG15-knockdown cells at various times post-infection with CSFV. LC3 is a widely used marker in autophagy research; during autophagy induction, LC-I is converted to LC3-II. As shown in Figures 5A and B, protein levels of LC3-II and accumulation of LC3 puncta were significantly reduced in CMV-ISG15 cells at both 12 hpi and 24 hpi. Similarly, the number of single- or double-membrane vesicles in the cytoplasm of CMV cells was higher than that in CMV-ISG15 cells (Figure 5C). As expected, knockdown of ISG15 obviously up-regulated both LC3-II expression and the fluorescent signal (Figures 5D and E). 3MA has been shown to inhibit autophagy via inhibition of autophagosome formation in PAMs during CSFV infection [19]. To further confirm that the antiviral action of ISG15 is mediated by direct inhibition of autophagy during CSFV infection, shN and shISG15 cells were treated with 3MA and infected with CSFV. As expected, knockdown of ISG15 expression failed to promote CSFV replication in the presence of 3MA (Figure 5F, G). These results demonstrated that ISG15 inhibits viral-induced autophagy to control CSFV replication. To determine whether ISG15 could also inhibit nonviral-induced autophagy, ISG15-overexpressing and ISG15-knockdown cells were treated with rapamycin, an autophagy inducer. The results showed that overexpression of ISG15 downregulated rapamycininduced autophagy (Figures 5H and I). In shISG15 cells, rapamycin induced a more obvious increase in LC3-II protein and LC3 puncta accumulation than in shN cells (Figures 5J and K). Taken together, these results suggested that ISG15 can inhibit the autophagy process induced by both CSFV and rapamycin.

\section{Autophagy protein BECN1 is ISGylated by ISG15}

To further understand how ISG15 influences the autophagic machinery during CSFV infection, BECN1 was overexpressed in ISG15-overexpressing cells. The LC3-II protein levels were evaluated in CSFV-infected cells that were co-transfected with CMV-ISG15, CMV and 3.1-BECN1, or 3.1. The LC3-II protein level and fluorescence signal were reduced by ISG15 but restored by BECN1 plasmid transfection, revealing that ISG15 regulates $\mathrm{BECN} 1$ to inhibit autophagy and CSFV replication (Figures 6A-C). To identify whether BECN1 is involved in ISGylation in PAMs during CSFV infection, BECN1 expression was evaluated in cells transfected with plasmids 3.1, 3.1-ISG15AA (expressing 


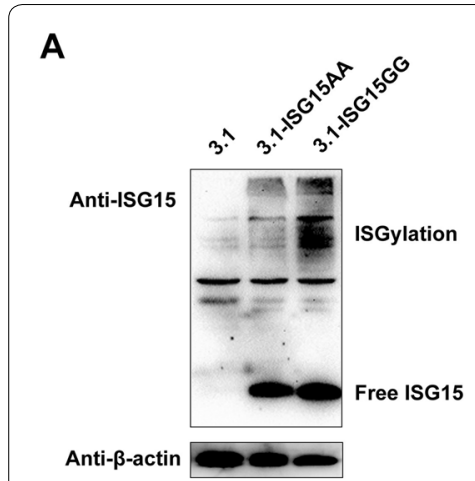

B
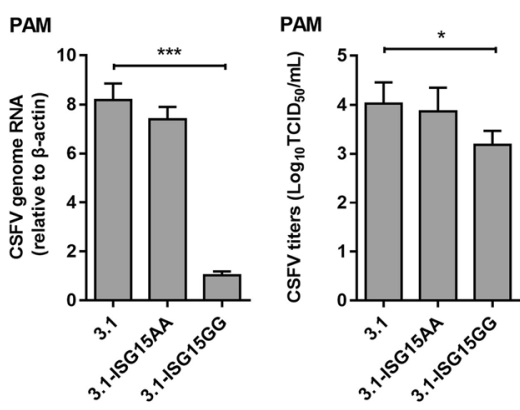

C

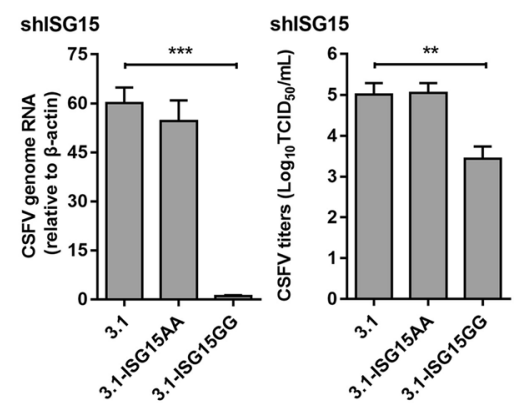

D

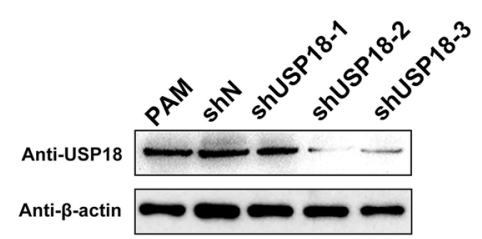

G

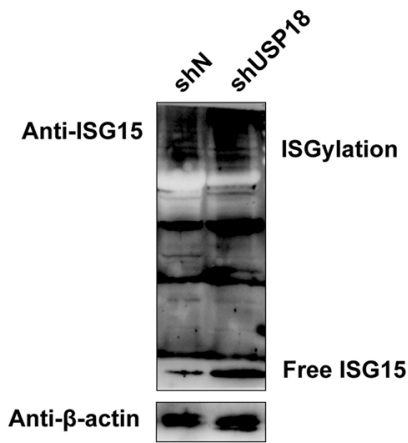

Anti- $\beta$ actin
E

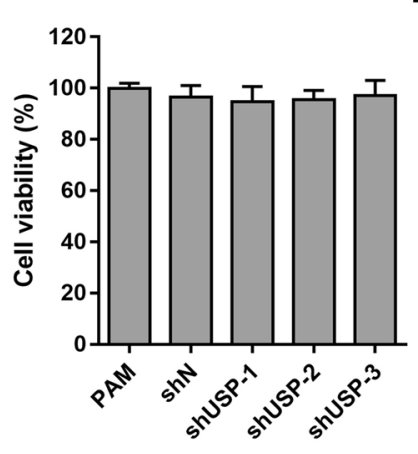

$\mathbf{F}$

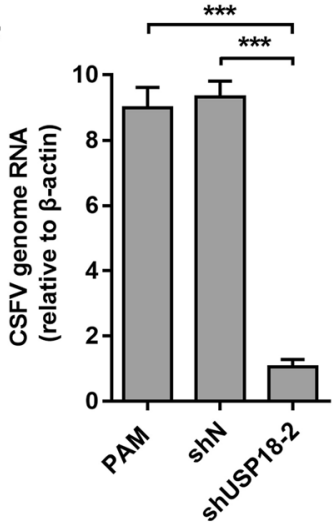

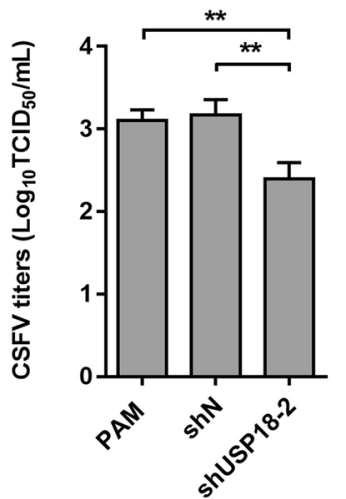

H

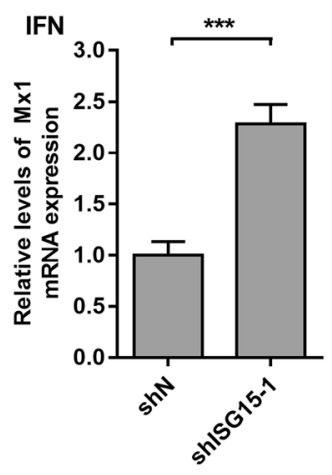

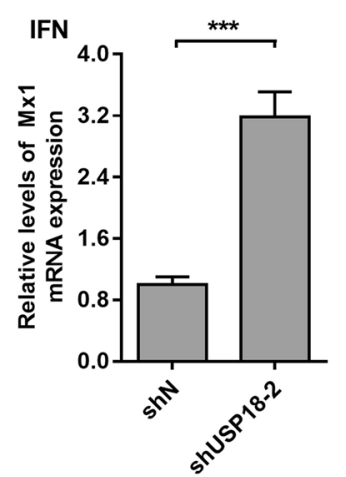

Figure 4 ISGylation is involved in the antiviral activity of ISG15 against CSFV. A Expression of ISGylated protein was detected by Western blot with an anti-ISG15 antibody. B, C Effects of ISGylation on CSFV replication. PAMs B and shISG15 cells $\mathbf{C}$ were transfected with 3.1, 3.1-ISG15AA, and 3.1-ISG15GG plasmid followed by CSFV infection for another $24 \mathrm{~h}(\mathrm{MOI}=1)$. CSFV replication was quantified by RT-qPCR and IFA, respectively. D, E PAMs with stable knockdown of USP18 were constructed by lentiviral transduction. D Expression of USP18 protein was detected by Western blot. E Cell viability with stable knockdown of USP18. ShN and shUSP18 cells were pretreated with IFN-a and then infected with CSFV (MOI=1). F CSFV replication was quantified by RT-qPCR and IFA, respectively. G Expression of ISGylated protein was detected by Western blot with an anti-ISG15 antibody. $\mathbf{H}$ Mxl expression was analyzed by RT-qPCR. Data (B, C, E, F, and $\mathbf{H})$ represent the mean \pm SD of three independent experiments and were measured in technical duplicate. Comparisons between groups were calculated using Student's t-test. ${ }^{* *} P<0.01 ;{ }^{* *} P<0.001$

non-conjugative ISG15), or 3.1-ISG15GG (expressing wild-type ISG15) following CSFV infection. Interestingly, BECN1 expression in 3.1-ISG15AA-transfected cells was similar to that in 3.1-transfected cells, whereas some specific protein bands appeared only for cells transfected with 3.1-ISG15GG (Figure 6D). It has been proven that fusing ISG15 LRLRGG with the target protein can mimic the constitutively modified state of ISGylation [20-22]. BECN1 has been shown to have at least four alternative ISGylation sites at its $\mathrm{N}$-terminus 
A

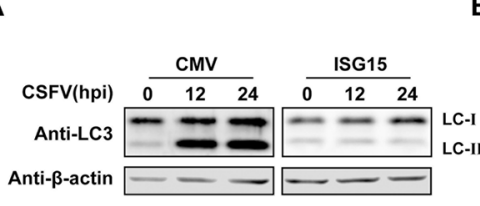

B

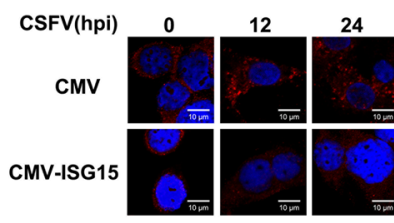

C
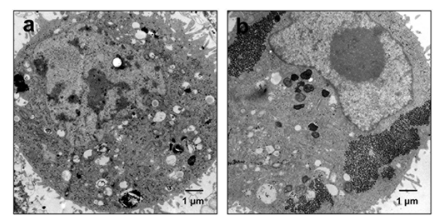

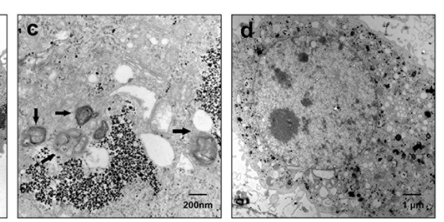

E

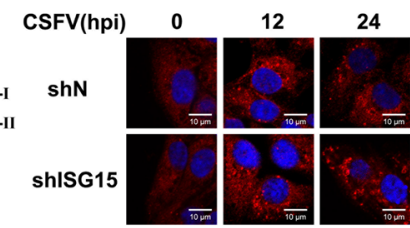

H
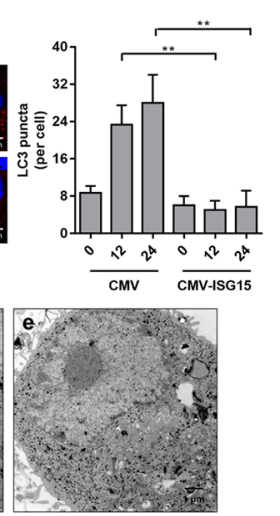

D

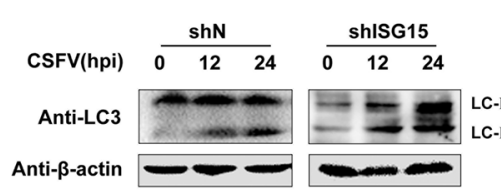

F

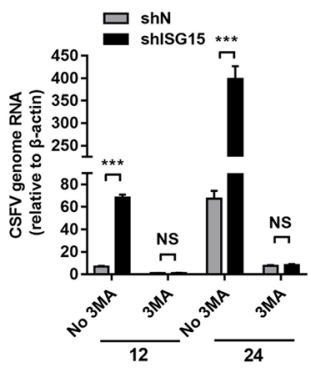

I

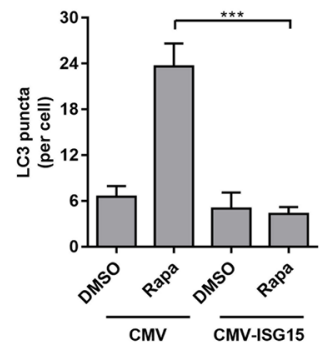

G

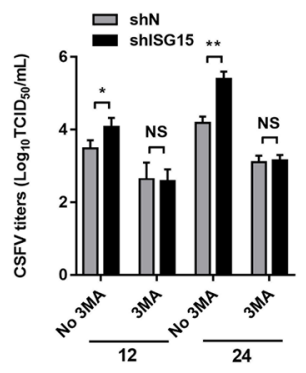

J

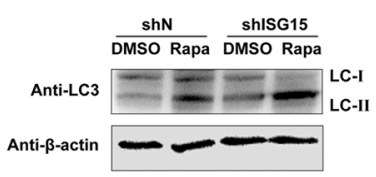

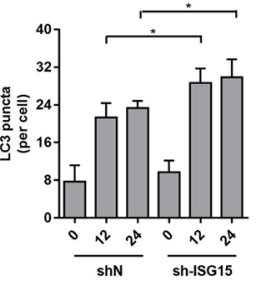

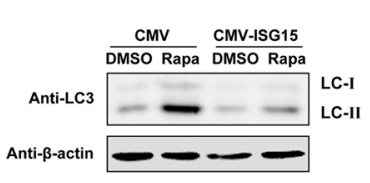

K

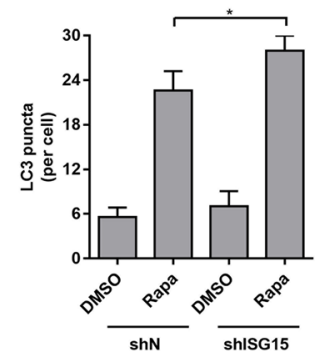

Figure 5 ISG15 inhibits autophagy, which is required for CSFV replication. CMV, CMV-ISG15, shN, and shISG15 cells were infected with CSFV $(M O I=1)$ for $12 \mathrm{~h}$ and $24 \mathrm{~h}$. LC3 protein $(\mathbf{A}, \mathbf{D})$ was analyzed by Western blot, and LC3 puncta (B, E) in PAMs were observed by confocal immunofluorescence microscopy. Cells were fixed and subjected to IFA by immunostaining with anti-LC3 antibody. Representative cells are shown. Scale bar $=10 \mu \mathrm{m}$. The number of LC3 puncta per cell was determined by image analysis. C Transmission electron microscopy images. CMV and CMV-ISG15 cells were mock-infected or infected with CSFV $(\mathrm{MOI}=1)$ for $24 \mathrm{~h}$ and studied by electron microscopy. Representative images of CMV cells (a), CSFV-infected CMV cells (b), magnified view of autophagosome-like vesicles (c), CMV-ISG15 cells (d), and CSFV-infected CMV-ISG15 cells (e) are shown. Scale bars $=1 \mu \mathrm{m}(\mathrm{a}, \mathrm{b}, \mathrm{d}$, and e) and $200 \mathrm{~nm}$ (c). Black arrows indicate the structures with the characteristics of autophagosomes. F, G ShN and shISG15 cells were pretreated with 3MA for $4 \mathrm{~h}$ after $1 \mathrm{~h}$ of virus adsorption, after which the cells were provided medium with $3 \mathrm{MA}$. After 24 h, CSFV replication was quantified by RT-qPCR and IFA, respectively. CMV and CMV-ISG15 cells (H and I) or shN and shISG15 cells (J, K) were treated with DMSO or rapamycin. Expression of $L C 3$ protein $(\mathbf{H}, \mathbf{J})$ and $L C 3$ puncta $(\mathbf{I}, \mathbf{K})$ in PAMs were quantified by image analysis. Data $(\mathbf{B}, \mathbf{E}, \mathbf{F}, \mathbf{G}, \mathbf{I}$, and $\mathbf{K}$ ) represent the mean \pm SD of three independent experiments and were measured in technical duplicate. Comparisons between groups were calculated using Student's t-test. ${ }^{*} P<0.05 ;{ }^{* *} P<0.01 ;{ }^{* * *} P<0.001$ 


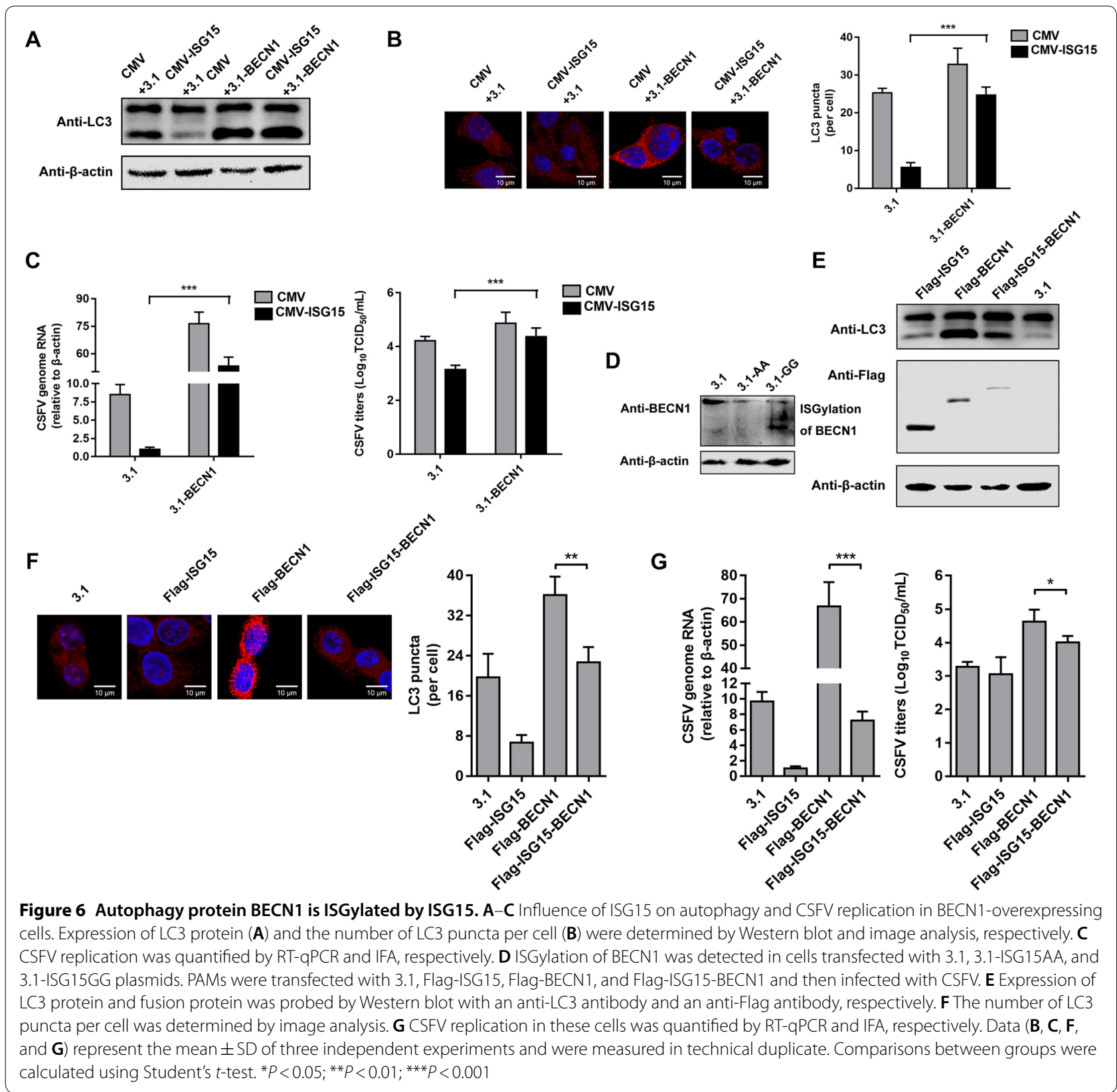

in $\mathrm{H} 4$ cells. Thus, we constructed Flag-ISG15-BECN1 recombinant plasmid in which LRLRGG of ISG15 was fused to the N-terminus of BECN1 to mimic BECN1 ISGylation. As shown in Figures 6E-G, LC3-II levels, the numbers of LC3 puncta, CSFV genomic RNA levels, and viral titers in Flag-ISG15-BECN1-expressing cells were obviously reduced compared with those in normal BECN1-expressing cells and PAMs transfected with 3.1 as a negative control. These results indicated that BECN1 ISGylated by ISG15 is unable to perform its function in autophagy and fails to promote CSFV replication.

\section{BECN1 interacts with the ISG15 E3 ligase HERC5}

It is well known that ISG15 conjugation to protein substrates typically requires HERC5, which functions as an ISG15 E3 ligase to promote ISGylation [18]. Thus, we investigated whether a physical interaction exists between these proteins. Co-IP assays were performed, and the plasmids Flag-BECN1 and Flag-RFP were 


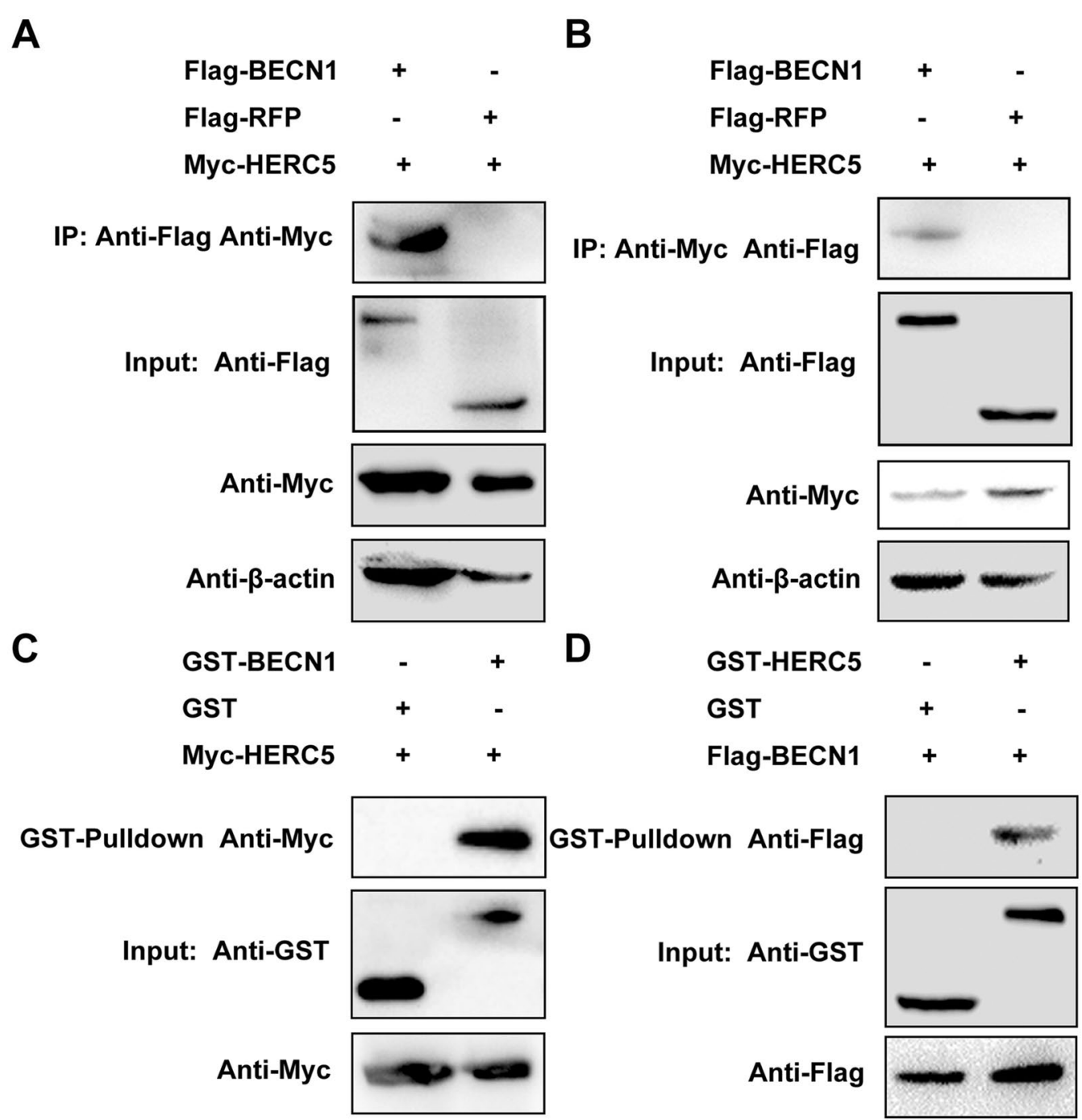

Figure 7 BECN1 interacts with the ISG15 E3 ligase HERC5. A PAMs were co-transfected with the plasmids Flag-BECN1 and Myc-HERC5 or Flag-RFP and Myc-HERC5 as a control. Cells were lysed and subjected to an IP assay with Anti-Flag M2 Affinity Gel. The precipitated proteins were detected by Western blot with an anti-Myc antibody. B PAMs were co-transfected with the plasmids Flag-BECN1 and Myc-HERC5 or Flag-RFP and Myc-HERC5 as a control. Cells were lysed and subjected to an IP assay with an anti-c-Myc immunoprecipitation kit. The precipitated proteins were analyzed by Western blot with an anti-Flag antibody. C GST and GST-BECN1 fusion proteins expressed in E. coli BL21 cells were conjugated with glutathione-Sepharose resin and incubated with lysate of HEK-293T cells overexpressing the Myc-HERC5 protein. The bound proteins were detected by Western blot with an anti-Myc antibody. D GST and GST-HERC5 fusion proteins expressed in E. coli were conjugated with glutathione-Sepharose resin and incubated with lysate of HEK-293T cells overexpressing the Flag-BECN1 protein. The bound proteins were detected by Western blot with an anti-Flag antibody

transfected into PAMs to detect Myc-tagged HERC5 protein. The result showed that Myc-HERC5 interacted with Flag-BECN1 but not Flag-RFP (Figure 7A). In the reciprocal co-IP assay, Flag-BECN1 was co-precipitated with Myc-HERC5, but the negative control Flag-RFP was not (Figure $7 \mathrm{~B}$ ). These findings demonstrated that BECN1 interacts with HERC5 in PAMs.

The GST pull-down assay was performed to further confirm the interaction between BECN1 and HERC5.
GST-BECN1 and GST were expressed in E. coli BL21 cells, and Myc-HERC5 was expressed in HEK-293T cells. Western blot assays showed that Myc-HERC5 was captured by GST-BECN1 (Figure 7C). Furthermore, GST-HERC5 or GST was expressed in bacteria, and cell lysates containing Flag-BECN1 were analyzed. FlagBECN1 was detected in GST-HERC5 complexes but not in GST complexes (Figure 7D). These results demonstrated that BECN1 interacts with HERC5 in vitro. 


\section{Discussion}

ISG15 has been proposed as an efficient host effector that defends against viral infections including that of human cytomegalovirus [17], human immune deficiency virus (HIV) [23], West Nile virus [24], and porcine reproductive and respiratory syncytial virus [25]. Macrophages are at the frontline of defense against pathogenic microorganisms. In our study, we provided the first strong evidence that ISG15 is an antiviral factor against CSFV in immune cells. Not only IFN-induced ISG15 but also overexpressed ISG15 alone obviously inhibited CSFV replication in PAMs (Figures 1 and 2). The expression of many ISGs is induced following type I IFN treatment. However, some of them exert independent antiviral functions that may affect CSFV replication $[6,7,9]$. This knowledge helps to explain why knockdown of ISG15 notably enhanced CSFV replication but did not totally neutralize the anti-CSFV activity of IFN- $\alpha$ (Figures 2D, E). In this study, ISG15 was significantly increased during CSFV infection in PAMs. Similar experiments were performed in other cell types with consistent results. A recent study reported that IRF1 mediates the increase in ISG15 expression during CSFV infection in PK-15 cells. In this process, IRF1 interacts with nucleotides -487 to -325 , which are located in the $5^{\prime}$ flanking region of the ISG15 gene, following stimulation with dsRNA or CSFV [26]. Therefore, we speculated that ISG15 might be induced by CSFV in an IFN-independent manner in PAMs, which will be further verified in future studies. Similar results were obtained in research of Vaccine-Cstrain; the ISG15 pathway was activated in response to Vaccine C-strain and ISG15 played a role in the rapid and early protection conferred by Vaccine C-strain [27]. In further research, we will test ISG15 effects on more CSFV genotypes to perfect the mechanisms of ISG15.

In addition to the unconjugated form of ISG15, which may be released from IFN-induced cells to the environment, the conjugated form of ISG15 can conjugate to numerous host and viral proteins [28]. With respect to other flaviviruses, overexpression of ISG15 significantly inhibits replication of Japanese encephalitis virus and West Nile virus via protein ISGylation [24, 29]. In this study, we used two methods to demonstrate that ISGylation was involved in the inhibition of CSFV replication mediated by ISG15. The C-terminal LRLRGG domain of ISG15 has been proven to be required for ISG15 conjugate formation [30,31]. Firstly, we constructed a mutant of ISG15. Counteraction of ISGylation by mutation of the conjugation site (LRLRGG) to LRLRAA significantly increased CSFV replication, indicating that ISGylation plays an important role in limiting CSFV replication. The induced ISGylation was cleaved especially by USP18, which belongs to the family of deubiquitinating enzymes
[32]. Secondly, we constructed a stable USP18-knockdown cell line. The ISGylation levels were increased and proliferation of CSFV was suppressed by downregulating USP18 expression, suggesting that inhibition of CSFV by ISG15 depends on ISGylation. Interestingly, knockdown of USP18 enhanced expression of ISG mRNA in this experiment. We found that knockdown of both ISG15 and USP18 upregulated ISG expression and exerted opposite effects on CSFV. In ISG15-knockdown cells pretreated with IFN- $\alpha$, the replication of CSFV was upregulated, even though ISG expression was upregulated. With regard to CSFV inhibition, the effect of ISGylation of ISG15 was predominant compared with the effect of other ISGs.

The E2 and NS5A proteins of CSFV are colocalized with autophagosome-like vesicles; autophagy provides vesicle membranes to promote CSFV replication and maturity in PK-15 cells and PAMs [14, 33]. Our research showed that ISG15 has a negative effect on both CSFVinduced and rapamycin-induced autophagy. When we inhibited autophagy levels, we found that downregulated ISG15 expression did not promote CSFV replication.

BECN1 is a key initiator of autophagy and associates with PtdIns3K, which mediates biogenesis and the dynamics of subcellular membranes and is involved in the autophagic process $[34,35]$. In H4 cells and HepG2 cells, BECN1 has been identified as a target protein of ISGylation. In addition, ISGylation of BECN1 inhibits PI3KC3 complex activation, which plays a pivotal role in autophagy, and USP18 positively regulates autophagy by promoting de-ISGylation of BECN1 [36, 37]. Nonetheless, in swine cells, the relationship between ISG15 and autophagy has not been reported. We showed that BECN1 was ISGylated during the antiviral action of ISG15 against CSFV. This modification affected the autophagy pathway, leading to the inhibition of CSFV replication. We further validated this result using fusion Flag-ISG15-BECN1 protein. Such proteins have been confirmed to mimic the ISG15-modified state of a protein and are especially useful when the modification site is near the terminus of the protein [18, 21, 22]. Flag-ISG15BECN1 expression was not able to initiate autophagy and induce CSFV replication like BECN1. These results are also consistent with our hypothesis that modification of autophagy by ISGylation of BECN1 impedes CSFV utilization of autophagy for self-production.

HERC5 functions as an ISG15 E3 ligase to promote ISGylation [18]. Recent studies support the contention that ISGylation of target proteins is regulated by HERC5. HERC5 especially catalyzes the ISGylation of ISG15 onto IRF3, which regulates innate antiviral activity in Sendai virus infection [38]. ISGylation of the target protein Parkin is specifically mediated by the ISG15 E3 ligase 
HERC5 [39]. As shown in Figure 7, HERC5 appeared to target BECN1, indicating that HERC5 may mediate covalent ISG15 conjugation to BECN1 in PAMs. ISG15 may also regulate autophagy-independent activities whose contributions to CSFV proliferation remain to be investigated. However, our current findings highlight the functional importance of ISG15 with regard to the antiviral activity of IFN and confirm that ISG15 functions as an anti-CSFV effector by regulating autophagy.

\begin{abstract}
Abbreviations
3MA: 3-methyladenine; BECN1: beclin-1; Co-IP: co-immunoprecipitation; CSF: classical swine fever; CSFV: classical swine fever virus; DMEM: Dulbecco's minimal essential medium; FBS: fetal bovine serum; FITC: fluorescein isothiocyanate; GST: glutathione S-transferase; HERC5: HECT and RLD domain containing E3 ubiquitin protein ligase 5; hpi: hour post-infection; IFA: indirect immunofluorescence assay; IFNs: interferons; ISGs: interferon-stimulated genes; MAb: monoclonal antibody; MOI: multiplicity of infection; PAb: polyclonal antibody; PAMs: porcine alveolar macrophages; PBS: phosphate-buffered saline; PCR: polymerase chain reaction; RT-qPCR: real-time quantitative polymerase chain reaction; SD: standard deviation; SDS-PAGE: sodium dodecyl sulfate-polyacrylamide gel electrophoresis; shRNA: short hairpin RNA; shN: scrambled control shRNA; TBST:Tris-buffered saline containing 0.5\% Tween 20; $\mathrm{TCID}_{50}: 50 \%$ tissue culture infectious dose; TEM: transmission electron microscopy; USP18: ubiquitin-specific protease 18.
\end{abstract}

\section{Acknowledgements}

This study was supported by the National Natural Science Foundation of China (No. 31472210).

\section{Authors' contributions}

Conceptualization, CL and YZ; Methodology, HZ and WD; Software, YE; Project administration, $\mathrm{CL}$ and $\mathrm{HL}$; Formal analysis, JL; Data curation, $\mathrm{CL}$ and WD; Writing — original draft preparation, KG; Writing —review and editing, CL; Supervision, YZ. All authors read and approved the final manuscript.

\section{Funding}

This study was supported by the National Natural Science Foundation of China (No. 31472210).

\section{Availability of data and materials}

All data generated or analyzed during this study are included in this published article.

\section{Competing interests}

The authors declare that they have no competing interests.

\section{Author details}

${ }^{1}$ College of Veterinary Medicine, Northwest A\&F University, Yangling, Shaanxi, China. ${ }^{2}$ Tianjin Animal Husbandry and Veterinary Research Institute, Tianjin, China. ${ }^{3}$ Henan University of Animal Husbandry and Economy, Zhengzhou, China. ${ }^{4}$ School of Nursing, Southwest Medical University, Luzhou, Sichuan, China.

Received: 29 September 2019 Accepted: 31 January 2020

Published online: 24 February 2020

\section{References}

1. Luo Y, Ji S, Liu Y, Lei JL, Xia SL, Wang Y, Du ML, Shao L, Meng XY, Zhou M, Sun Y, Qiu HJ (2017) Isolation and characterization of a moderately virulent classical swine fever virus emerging in China. Transbound Emerg Dis 64:1848-1857
2. Lv H, Dong W, Cao Z, Li X, Wang J, Qian G, Lv Q, Wang C, Guo K, Zhang $Y$ (2017) TRAF6 is a novel NS3-interacting protein that inhibits classical swine fever virus replication. Sci Rep 7:6737

3. Schneider WM, Chevillotte MD, Rice CM (2014) Interferon-stimulated genes: a complex web of host defenses. Annu Rev Immunol 32:513-545

4. Schoggins JW, Wilson SJ, Panis M, Murphy MY, Jones CT, Bieniasz P, Rice CM (2011) A diverse range of gene products are effectors of the type I interferon antiviral response. Nature 472:481-485

5. Fernandez-Sainz I, Ramanathan P, O'Donnell V, Diaz-San Segundo F, Velazquez-Salinas L, Sturza DF, Zhu J, de los Santos T, Borca MV (2015) Treatment with interferon-alpha delays disease in swine infected with a highly virulent CSFV strain. Virology 483:284-290

6. Li LF, Yu J, Li Y, Wang J, Li S, Zhang L, Xia SL, Yang Q, Wang X, Yu S, Luo Y, Sun Y, Zhu Y, Munir M, Qiu HJ (2016) Guanylate-binding protein 1, an interferon-induced GTPase, exerts an antiviral activity against classical swine fever virus depending on its GTPase activity. J Virol 90:4412-4426

7. Li LF, Yu J, Zhang Y, Yang Q, Li Y, Zhang L, Wang J, Li S, Luo Y, Sun Y, Qiu HJ (2017) Interferon-inducible oligoadenylate synthetase-like protein acts as an antiviral effector against classical swine fever virus via the MDA5mediated type I interferon-signaling pathway. J Virol 91:01514-01516

8. Zhao Y, Pang D, Wang T, Yang X, Wu R, Ren L, Yuan T, Huang Y, Ouyang H (2011) Human MxA protein inhibits the replication of classical swine fever virus. Virus Res 156:151-155

9. Li W, Mao L, Cao Y, Zhou B, Yang L, Han L, Hao F, Lin T, Zhang W, Jiang J (2017) Porcine Viperin protein inhibits the replication of classical swine fever virus (CSFV) in vitro. Virol I 14:202

10. Li C, Zheng H, Wang Y, Dong W, Liu Y, Zhang L, Zhang Y (2019) Antiviral role of IFITM proteins in classical swine fever virus infection. Viruses 11:E126

11. Yan Q, Yang H, Yang D, Zhao B, Ouyang Z, Liu Z, Fan N, Ouyang H, Gu W, Lai $L$ (2014) Production of transgenic pigs over-expressing the antiviral gene Mx1. Cell Regen (Lond) 3:11

12. Chang C, Su H, Zhang D, Wang Y, Shen Q, Liu B, Huang R, Zhou T, Peng C, Wong CC, Shen HM, Lippincott-Schwartz J, Liu W (2015) AMPK-dependent phosphorylation of GAPDH triggers Sirt1 activation and is necessary for autophagy upon glucose starvation. Mol Cell 60:930-940

13. Parzych KR, Klionsky DJ (2014) An overview of autophagy: morphology, mechanism, and regulation. Antioxid Redox Signal 20:460-473

14. Pei J, Zhao M, Ye Z, Gou H, Wang J, Yi L, Dong X, Liu W, Luo Y, Liao M, Chen $J$ (2014) Autophagy enhances the replication of classical swine fever virus in vitro. Autophagy 10:93-110

15. Wirawan E, Lippens S, Vanden Berghe T, Romagnoli A, Fimia GM, Piacentini $\mathrm{M}$, Vandenabeele $\mathrm{P}$ (2012) Beclin1: a role in membrane dynamics and beyond. Autophagy 8:6-17

16. Morales DJ, Lenschow DJ (2013) The antiviral activities of ISG15. J Mol Biol 425:4995-5008

17. Kim YJ, Kim ET, Kim YE, Lee MK, Kwon KM, Kim Kl, Stamminger T, Ahn $\mathrm{JH}$ (2016) Consecutive inhibition of ISG15 expression and ISGylation by cytomegalovirus regulators. PLoS Pathog 12:e1005850

18. Wong JJ, Pung YF, Sze NS, Chin KC (2006) HERC5 is an IFN-induced HECTtype $\mathrm{E} 3$ protein ligase that mediates type I IFN-induced ISGylation of protein targets. Proc Natl Acad Sci U S A 103:10735-10740

19. Wu YT, Tan HL, Shui G, Bauvy C, Huang Q, Wenk MR, Ong CN, Codogno P, Shen HM (2010) Dual role of 3-methyladenine in modulation of autophagy via different temporal patterns of inhibition on class I and III phosphoinositide 3-kinase. J Biol Chem 285:10850-10861

20. Okumura F, Okumura AJ, Uematsu K, Hatakeyama S, Zhang DE, Kamura T (2013) Activation of double-stranded RNA-activated protein kinase (PKR) by interferon-stimulated gene 15 (ISG15) modification down-regulates protein translation. J Biol Chem 288:2839-2847

21. Okumura F, Zou W, Zhang DE (2007) ISG15 modification of the elF4E cognate 4EHP enhances cap structure-binding activity of 4EHP. Genes Dev 21:255-260

22. Ross S, Best JL, Zon LI, Gill G (2002) SUMO-1 modification represses Sp3 transcriptional activation and modulates its subnuclear localization. Mol Cell 10:831-842

23. Okumura A, Lu G, Pitha-Rowe I, Pitha PM (2006) Innate antiviral response targets HIV-1 release by the induction of ubiquitin-like protein ISG15. Proc Natl Acad Sci USA 103:1440-1445

24. Dai J, Pan W, Wang P (2011) ISG15 facilitates cellular antiviral response to dengue and west nile virus infection in vitro. Virol J 8:468 
25. Sun Z, Li Y, Ransburgh R, Snijder EJ, Fang Y (2012) Nonstructural protein 2 of porcine reproductive and respiratory syndrome virus inhibits the antiviral function of interferon-stimulated gene 15. J Virol 86:3839-3850

26. Li XQ, Li XN, Liang JJ, Cai XB, Tao Q, Li YX, Qin Q, Xu SP, Luo TR (2018) IRF1 up-regulates isg 15 gene expression in dsRNA stimulation or CSFV infection by targeting nucleotides -487 to -325 in the $5^{\prime}$ flanking region. Mol Immunol 94:153-165

27. McCarthy RR, Everett HE, Graham SP, Steinbach F, Crooke HR (2019) Head start immunity: characterizing the early protection of $C$ strain vaccine against subsequent classical swine fever virus infection. Front Immunol 10:1584

28. Skaug B, Chen ZJ (2010) Emerging role of ISG15 in antiviral immunity. Cell 143:187-190

29. Hsiao NW, Chen JW, Yang TC, Orloff GM, Wu YY, Lai CH, Lan YC, Lin CW (2010) ISG15 over-expression inhibits replication of the Japanese encephalitis virus in human medulloblastoma cells. Antiviral Res 85:504-511

30. Lenschow DJ, Giannakopoulos NV, Gunn LJ, Johnston C, O'Guin AK, Schmidt RE, Levine B, Virgin HW (2005) Identification of interferonstimulated gene 15 as an antiviral molecule during Sindbis virus infection in vivo. J Virol 79:13974-13983

31. Gonzalez-Sanz R, Mata M, Bermejo-Martin J, Alvarez A, Cortijo J, Melero JA, Martinez I (2016) ISG15 is upregulated in respiratory syncytial virus infection and reduces virus growth through protein ISGylation. J Virol 90:3428-3438

32. Basters A, Knobeloch KP, Fritz G (2018) How USP18 deals with ISG15modified proteins: structural basis for the specificity of the protease. FEBS J 285:1024-1029
33. Ji W, Guo Z, Ding NZ, He CQ (2015) Studying classical swine fever virus: making the best of a bad virus. Virus Res 197:35-47

34. Pattingre S, Tassa A, Qu X, Garuti R, Liang XH, Mizushima N, Packer M, Schneider MD, Levine B (2005) Bcl-2 antiapoptotic proteins inhibit Beclin 1-dependent autophagy. Cell 122:927-939

35. Glover K, Li Y, Mukhopadhyay S, Leuthner Z, Chakravarthy S, Colbert CL (2017) Structural transitions in conserved, ordered Beclin 1 domains essential to regulating autophagy. J Biol Chem 292:16235-16248

36. Xu D, Zhang T, Xiao J, Zhu K, Wei R, Wu Z, Meng H, Li Y, Yuan J (2015) Modification of BECN1 by ISG15 plays a crucial role in autophagy regulation by type I IFN/interferon. Autophagy 11:617-628

37. Wang X, Li C, Zhou L, Zhang N, Wang X, Ge X, Guo X, Yang H (2014) Porcine reproductive and respiratory syndrome virus counteracts the porcine intrinsic virus restriction factors-IFITM1 and Tetherin in MARC-145 cells. Virus Res 191:92-100

38. Shi HX, Yang K, Liu X, Liu XY, Wei B, Shan YF, Zhu LH, Wang C (2010) Positive regulation of interferon regulatory factor 3 activation by Herc 5 via ISG15 modification. Mol Cell Biol 30:2424-2436

39. Im E, Yoo L, Hyun M, Shin WH, Chung KC (2016) Covalent ISG15 conjugation positively regulates the ubiquitin E3 ligase activity of parkin. Open Biol 6:160193

\section{Publisher's Note}

Springer Nature remains neutral with regard to jurisdictional claims in published maps and institutional affiliations.
Ready to submit your research? Choose BMC and benefit from:

- fast, convenient online submission

- thorough peer review by experienced researchers in your field

- rapid publication on acceptance

- support for research data, including large and complex data types

- gold Open Access which fosters wider collaboration and increased citations

- maximum visibility for your research: over $100 \mathrm{M}$ website views per year

At BMC, research is always in progress.

Learn more biomedcentral.com/submissions 